\title{
Reflujo faringolaríngeo: Revisión bibliográfica
}

\author{
Pharyngolaryngeal reflux: A literature review
}

Rodolfo Nazar $\mathrm{S}^{1}$, Juan Fullá $0^{2}$, Mํㅡㄹ Antonieta Varas P², Alfredo Naser G1.

\section{RESUMEN}

日 reflujo faringolaríngeo (RR) es un síndrome que se asocia a una constelación de síntomas. Entre ellos los más frecuentemente descritos son la disfonía, la fatiga vocal, el globus faríngeo, la tos crónica y la carraspera.

Se realizó una búsqueda exhaustiva en bases de datos internacionales y nacionales en relación a $\mathrm{R} R$. Se utilizaron palabras clave como globus, carraspera, laringitis, disfonía y reflujo gastroesofágico. Se obtuvieron alrededor de 35 referencias en relación al tema estudiado, siendo la mayoría publicadas con posterioridad al año 2000.

En relación a su etiología, se cree que el flujo retrógrado de contenido gástrico hacia la faringe sería el hecho más importante para su génesis, pero recientemente se ha demostrado que si bien este hecho patológico es fundamental en la producción de los síntomas, existen factores adicionales, capaces de perpetuarlo, permitiendo considerar al $R R$ como una entidad diferente del reflujo gastroesofágico (RCE).

Palabras clave: Reflujo faringolaríngeo, reflujo gastroesofágico.

\begin{abstract}
Pharyngolaryngeal reflux (RR) is a syndrome associated to a variety of symptoms. Among them, the most frequently described are dysphonia, vocal fatigue, pharyngeal globus, chronic coughing and throat clearing.

An exhaustive search of international and national databases was performed. Gobus, throat clearing, laryngitis and gastroesophageal reflux were used as keywords. Some 35 search-related references were retrieved, of which most were published from 2000 to date. Respect to the etiology of pharyngolaryngeal reflux, the current view is that gastric content reflux to the pharynx is the most important factor, but recently it has been demonstrated that, although this pathological trait is pivotal for symptoms appearance, there are additional factors that could perpetuate the symptoms in time, which contribute to consider RA as a different entity from gastroesophageal reflux (RGE).
\end{abstract}

Key words. Pharyngolaryngeal reflux, gastroesophageal reflux.

1 Médico Otorrinolaringólogo, Hospital Clínico de la Universidad de Chile.

2 Interno de Medicina, sede Norte de la Universidad de Chile. 


\section{INTRODUCCIÓN}

El reflujo faringolaríngeo (RFL) es un síndrome que se presenta con múltiples manifestaciones clínicas, como laringitis, disfonía, fatiga vocal, globus, carraspeo excesivo, tos crónica y disfagia leve. Dichos síntomas son causados por un flujo gástrico, que en forma retrógrada alcanza la laringofaringe ${ }^{1}$. Este hecho es compartido por el reflujo gastroesofágico (RGE), el cual se define como el ascenso de contenido estomacal hacia el esófago, presentándose sin náuseas 0 vómitos ${ }^{2}$. Si bien ambas patologías comparten el mismo punto de partida, son consideradas entidades distintas, cuyos síntomas y necesidad de tratamiento son diferentes. La suposición de este hecho, se basa en estudios realizados en sujetos con síntomas de RFL, los cuales, al ser interrogados, no presentaron los síntomas clásicos de RGE. Además, se observó que al ser sometidos a una endoscopía digestiva alta, su resultado era informado dentro de límites normales. El primero en describir la existencia del RFL fue James Koufman en el año 1988, el cual surgió tras la necesidad de definir una entidad patológica, la cual se pensaba estaba relacionada con el $\mathrm{RGE}^{3}$. Con el correr de los años, esta entidad se ha hecho más conocida, generándose un gran número de publicaciones que intentan explicar el trastorno fisiopatológico de este síndrome. La importancia del RFL radica en que se presenta en hasta el $10 \%$ de los pacientes que acuden a la consulta otorrinolaringológica. Además, se ha observado que en más del $50 \%$ de los pacientes con disfonía, existen signos de la existencia de una enfermedad por reflujo subyacente ${ }^{4}$.

\section{EPIDEMIOLOGÍA}

Existen pocos datos acerca de la prevalencia de RFL. Los resultados de un estudio en el cual participaron 105 pacientes quienes fueron evaluados mediante laringoscopía, informaron que el $86 \%$ de ellos presentaba algún signo de reflujo ${ }^{7}$. Por otra parte, en un metanálisis, en el cual se incluyeron estudios en los cuales se utilizó una sonda de medición de pH, encontró que entre el $10 \%$ y el $60 \%(30 \%$ en promedio) de los pacientes del grupo control, presentaban algún grado de reflujo.

En el año 2007, Vavricka y cols. presentaron los resultados de un estudio, a través del cual se evaluó el valor diagnóstico de la presencia de hallazgos sugerentes de RFL, en pacientes con RGE. En total, fueron evaluados 1.209 pacientes, quienes posteriormente fueron ingresados a un protocolo estructurado, a través del cual se les sometió a una evaluación mediante una endoscopía digestiva alta de rutina ${ }^{8}$. Durante el procedimiento, además, se llevó a cabo una cuidadosa evaluación laringofaríngea, la cual fue registrada en un video. Dicho registro fue revisado aleatoriamente por uno de tres gastroenterólogos y un otorrinolaringólogo. El número final de pacientes incluidos fue de 132 pacientes. Todos ellos cumplían con ciertas características como no encontrarse en tratamiento con inhibidores de la bomba de protones, y presentar hallazgos compatibles con una esofagitis erosiva (Savary Miller ${ }^{1-4}$ ). Los resultados fueron comparados con un grupo control de igual número de pacientes, todos ellos sin síntomas de RGE, no fumadores y con el antecedente de haber utilizado inhibidores de la bomba de protones. Además se exigió una evaluación mediante endoscopía digestiva alta, la cual debía ser informada dentro de límites normales.

Tras la realización del estudio, se encontraron signos de reflujo laringofaríngeo con una incidencia similar en ambos grupos ( $44 \%$ vs $37 \%$ ). Dentro de los hallazgos descritos, se incluyeron: el engrosamiento de las aritenoides, el edema de la pared medial aritenoídea, una pared posterior faríngea con aspecto de empedrado y edema de la pared posterior del cricoides. El único signo que fue encontrado con mayor frecuencia en pacientes con RGE, fue el eritema de la pared posterior de la faringe.

\section{ETIOLOGÍA}

Tanto la etiología del RGE, como la del RFL se deben a factores multifactoriales. Dentro de estos se incluyen: una disfunción del esfínter esofágico, el tiempo de exposición al material refluido y el nivel de sensibilidad del tejido. Anteriormente se ha demostrado una mayor sensibilidad del epitelio laríngeo, que el epitelio esofágico, al reflujo de contenido gástrico. Sólo bastan tres episodios de $\mathrm{RFL}$, con pH inferior a 4 a la semana, para que se produzca daño, a diferencia del RGE que requiere del orden de 50 episodios semanales para producir 
algún grado de daño ${ }^{7}$. La explicación de esta mayor sensibilidad epitelial laríngea permanece aún poco clara, pero se cree que la presencia de un $\mathrm{pH}$ bajo a nivel de la laringe, acompañado de la presencia de pepsina, generaría una reacción, que aumentaría la producción de proteínas de estrés, afectando la reparación epitelial local, provocando el daño antes descrito. Esto permitiría que mínimas cantidades de material refluido provoquen daño, a diferencia de lo que ocurre en el epitelio esofágico.

\section{FISIOPATOLOGÍA}

Existen cuatro barreras fisiológicas que protegen la vía aérea superior, del daño provocado por el reflujo $0^{4,12}$. Estas son el esfínter esofágico superior e inferior, la mucosa esofágica y las ondas motoras del esófago (clearenceesofágico). Cuando alguna de estas barreras falla, el epitelio respiratorio de la laringe posterior pierde su función natural, la cual es barrer el mucus desde el árbol traqueobronquial, provocando acumulación de éste. Esta situación produce sensación de descarga posterior y provoca carraspeo laríngeo. Por otro lado el material refluido provoca tos $y$ laringoespasmo debido a que la sensibilidad de los terminales nerviosos de la laringe se regula a través de la inflamación local. La combinación de todos estos factores es capaz de producir edema, úlceras por contacto y granulomas, los cuales son responsables de otros síntomas relacionados al RFL como la voz ronca, el globus faríngeo y la odinofagia.

Estudios recientes sugieren que el tejido laríngeo estaría protegido de la acción del ácido, a través de la regulación del pH local, mediante la acción de una anhidrasa carbónica ubicada en la laringe posterior ${ }^{11}$. Su acción la llevaría a cabo mediante la hidratación de dióxido de carbono, desviando la reacción hacia la producción de bicarbonato, protegiendo de esta forma a la mucosa.

En forma más específica, se ha reportado la existencia de una isoenzima III de la anhidrasa carbónica, la cual se encuentra en altos niveles en un epitelio laríngeo normal. Esta enzima estaría expresada en menor frecuencia, llegando incluso a estar ausente hasta en el $64 \%$ de las biopsias practicadas, a pacientes con diagnóstico de RFL.

\section{DIAGNÓSTICO}

\section{Historia clínica}

Como parte de la clínica es importante recalcar el significado que tiene la existencia de disfonía ${ }^{13}$. La presencia de este síntoma, se presenta como un antecedente importante, aún por sobre el antecedente mismo de laringitis. La razón de este hecho es debido a que este último síntoma, además de ser poco específico, puede obedecer a múltiples causas, que van desde procesos autolimitados, hasta el trauma vocal. Con respecto a la disfonía funcional esta es definida como la dificultad en la producción de la voz, presentándose ésta, en ausencia de una alteración de la mucosa laríngea ${ }^{3}$.

Koufman fue el primero en distinguir claramente el RFL del RGE, mediante un reporte combinado de 899 pacientes $^{3}$. Entre los que tenían RFL, $87 \%$ presentaba carraspera, en contraposición con los diagnosticados con RGE, quienes referían dicho síntoma en el $3 \%$ de los casos. Por otro lado en relación a la presencia de síntomas como pirosis, dicha manifestación se encontraba presente en el $83 \%$ de los pacientes con RGE, a diferencia de los pacientes con RFL quienes lo referían en el $20 \%$ de los casos. Una encuesta internacional de la asociación Broncoesofágica Americana, reveló que los síntomas de RFL más frecuentemente encontrados eran: carraspera (98\%), tos crónica $(97 \%)$, globus faríngeo (95\%) y la disfonía (95\%). Belafsky y cols. desarrollaron una herramienta útil para ayudar a los clínicos a evaluar la gravedad de los síntomas referidos por los pacientes ${ }^{8}$. Esta se basa en una escala de 0 a 5 puntos. Además se les pregunta a los pacientes por síntomas como disfonía o problemas vocales (1), carraspera (2), descarga nasal posterior (3), odinofagia (4), tos después de comer 0 con el decúbito (5), dificultad para respirar o sensación de ahogo (6), problemas 0 molestias para toser (7), sensación de cuerpo extraño en la laringe (8) y pirosis 0 ardor retroesternal, dolor torácico, indigestión o regurgitaciones (9). Dentro de los resultados, se encontró que la presencia de dichos síntomas en pacientes con RFL, y que además, no tenían como antecedente no haber recibido tratamiento, fue significativamente mayor, que en aquellos que sí lo recibieron (21,2 vs 11,6 p. 0,001), lo que además mostró que puntajes sobre 13 se consideran como 
un resultado anormal, con un intervalo de confianza por sobre el $95 \%$.

\section{Laringoscopía}

En general no se describen signos específicos laríngeos de inflamación, sin embargo sí existen ciertos hallazgos capaces de sugerir fuertemente la presencia de RFL. Dentro de éstos, el más frecuente es la llamada "laringitis posterior" (Figura 1), que corresponde al engrosamiento, enrojecimiento y edema de la zona posterior de la laringe ${ }^{13}$. La presencia de granulomas también ha sido asociado de forma importante. (65\%$74 \%$ ) en los casos confirmados de RFL, mediante la monitorización de $\mathrm{pH}^{15}$. El llamado "surco vocal" corresponde a un pseudo sulcus, frecuentemente ubicada en la zona intermedia del pliegue vocal. Esta última condición ha sido reportada en el $90 \%$ de los casos de Ios pacientes con RFL. Al realizar una comparación entre un grupo de 30 pacientes con RFL con otro grupo control del mismo número de sujetos, la sola presencia del surco al examen, aumenta la probabilidad de tener un test de $\mathrm{pH}$ positivo para RFL en 2,5 veces ${ }^{13}$. Por otro lado la sensibilidad y especificidad reportada para la presencia del surco en pacientes con RFL fue de $70 \%$ y $77 \%$ respectivamente, por lo que este hallazgo sigue siendo solamente sugerente de esta patología. Partiendo de la base que no existen hallazgos patognomónicos de RFL, Belafsky y cols., elaboraron una escala que evalúa 8 ítemes clínicos, con el fin de objetivar la severidad de los hallazgos laringoscópicos ${ }^{16}$. Dichos ítemes son evaluados del 0 al 4 e incluyen la presencia de: edema subglótico, obliteración ventricular, eritema e hiperemia, edema de la cuerda vocal, edema laríngeo difuso, hipertrofia de la comisura posterior y la presencia de granulomas. Esta escala parece ser útil tanto para el diagnóstico como para el tratamiento de los pacientes y permite asegurar con $95 \%$ de certeza que con un puntaje mayor o igual a 7 , se está frente a hallazgos propios de RFL.

\section{Confirmación del reflujo}

Existen 3 acercamientos para confirmar el diagnóstico Estos son la respuesta sintomática a la terapia médica empírica y conductual, la objetivación del daño de la mucosa mediante endoscopía (Figura 1) y la demostración de reflujo mediante estudios de monitoreo de $\mathrm{pH}$. A estos se pueden agregar otros estudios como radiografías, manometría esofágica, medición espectrofotométrica de reflujo biliar y biopsia de la mucosa. Debido a que una gran cantidad de pacientes responden satisfactoriamente a la terapia conductual y al tratamiento médico empírico, éste se presenta como un antecedente potente, orientándonos como una primera aproximación diagnóstica. La terapia empírica se basa principalmente en el uso de inhibidores de la bomba de protones por al menos 3 meses. Como parte del estudio se puede considerar incluir una evaluación endoscópica o mediante una laringoscopía rígida, en todos los casos en que exista sospecha de RFL.

En general se ha reportado que la endoscopía tiene un bajo rendimiento, pero una experiencia reciente de Poelmans y cols. muestra resultados diferentes ${ }^{17}$. Se estudió un grupo de pacientes, con síntomas de RFL como odinofagia, disfonía, tos no productiva y globus, todos con respuesta refractaria al tratamiento. Al analizar su endoscopía, ésta mostró un rendimiento destacado de $52,3 \%$ para las lesiones relacionadas con la presencia de reflujo (esofagitis erosiva y esófago de Barret) comparadas con $42,6 \%$ del grupo control, quienes relataban síntomas clásicos de reflujo, como pirosis y regurgitación ácida.

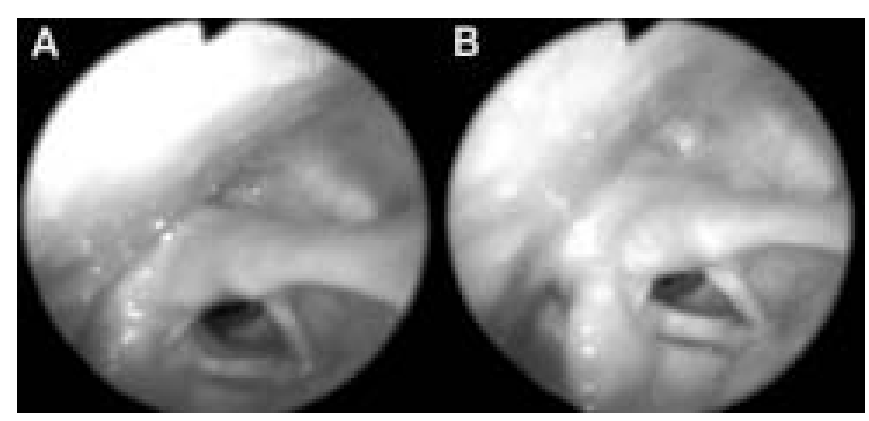

Figura 1. (A y B) Se aprecia la comisura posterior edematosa y de superficie irregular. 
En general los estudios con endoscopía digestiva alta (EDA) y la monitorización de $\mathrm{pH}$ durante 24 horas, han sido menos útiles en el estudio de pacientes con RFL que en los que con RGE. La EDA muestra lesiones esofágicas en el $50 \%$ de los pacientes con RGE, mientras que en los con RFL estos hallazgos se presentan en una cifra menor al $20 \%$. Para demostrar la existencia de RFL resulta más útil realizar un estudio ambulatorio multicanal de impedancia intraluminal y monitoreo de $\mathrm{pH}$.

En un estudio realizado por Vavricka y cols. en el año 2007, se estudió la prevalencia de cambios en el tejido de la región laringofaríngea, en pacientes con y sin hallazgos endoscópicos de RGE.

Los resultados de este estudio revelaron una alta prevalencia de cambios en esta zona, en pacientes a los cuales se les practicó una EDA. Hasta hace poco tiempo dichos signos habían sido considerados como patognomónicos de lesiones laringofaríngeas. Los resultados mostraron la misma prevalencia de lesiones, tanto en los pacientes con y sin RGE. Estos resultados sugieren que la mayoría de las lesiones laringofaríngeas que se suponen relacionadas a la presencia de ácido, tienen otras causas, diferentes a las relacionadas con el RGE.

\section{TRATAMIENTO}

\section{Educación y cambios conductuales}

Como parte de las medidas generales que se deben tomar una vez hecho el diagnóstico de RFL, está el educar a los pacientes con respecto a la naturaleza del problema y los cambios dietéticos y de comportamiento que deben realizar para disminuirlo. Dentro de los hábitos saludables se incluyen la pérdida de peso, dejar de fumar y evitar el alcohol. Los cambios en la dieta, incluyen, disminuir el consumo de chocolate, grasas, cítricos, bebidas carbonatadas, ciertos condimentos hechos en base a tomate, vino tinto, café y las comidas a media noche.

\section{Tratamiento farmacológico}

Existen 4 tipos de fármacos utilizados en el tratamiento del RFL. Entre ellos se encuentran: Ios inhibidores de la bomba de protones, los antagonistas del receptor $\mathrm{H} 2$, los agentes proquinéticos y los citoprotectores de la mucosa.
De estos el primer grupo constituye el pilar fundamental, aunque no están exentos de controversia en relación a su verdadera eficacia. Se recomienda realizar un mes de tratamiento con el fin de evaluar la respuesta al tratamiento. Si existe una respuesta satisfactoria debe evaluarse la suspensión del tratamiento, en caso contrario se sugiere iniciar estudios para confirmar el diagnóstico de RFL.

La ranitidina ha demostrado ser un inhibidor más potente de la secreción gástrica que la cimetidina, sin embargo ambos han demostrado un valor limitado para el tratamiento del RFL. Los agentes proquinéticos capaces de acelerar el clearenceesofágico y de incrementar la presión del esfínter esofágico inferior, han perdido credibilidad debido al reporte de efectos adversos con su uso, tales como arritmias ventriculares y diarrea.

Tegaserod es un agente proquinético que ha demostrado disminuir tanto el reflujo, como los episodios de relajaciones transitorias del esfínter esofágico inferior. Su uso en pacientes con RFL es efectivo cuando este se asocia a disquinesia esofágica. El Sucralfate es una sal de sucrosa polisulfatada, que podría ser útil como complemento en la protección de los daños producidos por la pepsina y el ácido sobre la mucosa. Los antiácidos sirven para aliviar los síntomas en el RGE pero no juegan ningún rol en el manejo del RFL.

En relación a la calidad de vida de los pacientes con RFL, Siupsinskiene y cols., llevaron a cabo un estudio para medir su impacto ${ }^{9}$. Este fue compuesto por un grupo de 100 pacientes con RFL y otro de 109 controles sanos. El grupo de pacientes con RFL fue sometido a una EDA y tratados con omeprazol por 3 meses. Dentro de dicho grupo 21 pacientes tuvieron esofagitis y 79 pacientes no, lo que permitió subdividir al grupo de pacientes con RFL en 2 subgrupos. La calidad de vida fue evaluada usando un índice de discapacidad vocal, una escala de depresión y ansiedad hospitalaria, la medición de la incapacidad para realizar actividades sociales y el sentimiento de bienestar general. Como conclusión se observó que la calidad de vida de los pacientes con RFL con 0 sin esofagitis se encuentra significativamente reducida en muchos aspectos. Este daño está más asociado con síntomas, más que con los hallazgos endoscópicos. El tratamiento con omeprazol mejoró significativamente la calidad de vida en ambos subgrupos de pacientes con RFL. 


\section{Cirugía}

Su uso es recomendado cuando el tratamiento médico falla, en pacientes en los cuales se ha demostrado un alto volumen de líquido refluido y una incompetencia del esfínter esofágico inferior. El procedimiento más frecuentemente utilizado es la funduplicatura, ya sea completa o parcial, prefiriéndose la vía laparoscópica para llevarlo a cabo ${ }^{18}$.

El objetivo del tratamiento quirúrgico es restablecer la competencia del esfínter esofágico inferior, y la evaluación de su resultado se lleva a cabo mediante la demostración en la disminución de los episodios de reflujo. Se han reportado excelentes resultados en el $85 \%$ a $95 \%$ de los casos de reflujo, pero sus resultados en pacientes con RFL no han sido igualmente de satisfactorios.

\section{BIBLIOGRAFÍA}

1. ForD C. Evaluation and Management of Laryngopharyngeal Reflux. JAMA 2005; 294(12); 1534-40.

2. Inzunza F, Brunetto B, Braghetto I, Olavarría C. Reflujo laringo gastroesofágico. Rev Hosp Cin Univ Chile 2001; 12(3): 192-6.

3. Koufman J. Laryngopharyngeal Reflux 2002: A New Paradigm of Airway Disease. Ear, Nose \& Throat Journal 2002; 81; 2-6.

4. KoufMAn J. The otolaryngologic manifestations of gastroesophageal reflux disease (GERD): a clinical investigation of 225 patients using ambulatory 24-hour pH monitoring and an experimental investigation of the role of acid and pepsin in the development of laryngeal injury. Laryngoscope. 1991; 101(4 pt 2 suppl 53): 1-78.

5. VaVRICKA SR, Storck CA, WILdI SM. Laryngopharyngeal Reflux: Beauty is in the Eye of the Beholder. Gastroenterology 2007; 133: 1379-81.

6. Groome M. Prevalence of Laryngopharyngeal Reflux in a Population With Gastroesophageal Reflux. Laryngoscope 2007, 117: 1424-8.

7. VavRICKA SR, Storck CA, WILDI SM. Limited Diagnostic Value of Laryngopharyngeal Lesios in
Patients With Gastroesophageal Reflux During Routine Upper Gastrointestinal Endoscopy. AmJ Gastroenterol 2007; 102: 716-22.

8. Belafsky PC, Postma GN, Koufman JA. Validity and reliability of the Reflux Symptom Index (RSI). J Voice. 2002; 16: 564-79.

9. Siupsinskiene N, Adamonis K, Toohill R. Quality of Life in Laryngopharyngeal Reflux Patients. Laryngoscope 2007; 117: 480-4.

10. Axford SE, Sharp N, Ross PE, et al. Cell biology of laryngeal epithelial defenses in health and disease: preliminary studies. Ann Otol Rhinol Laryngol. 2001; 110: 1099-1108.

11. Koufman J, Sataloff RT, Toohill R. Laryngopharyngeal reflux: consensus conference report. J Voice. 1996; 10: 215-6.

12. Belafsky PC, Postma GN, Koufman JA. The association between laryngeal pseudosulcus and laryngopharyngeal reflux. Oolaryngol Head Neck Surg 2002; 126: 649-52.

13. Ylitalo R, Lindestad PA, Ramel S. Symptoms, laryngeal findings, and 24-hour pH monitoring in patients with suspected gastroesophagopharyngeal reflux. Laryngoscope. 2001; 111: 1735-41.

14. Karkos Petros D, Yates Phillp D, Carding Paul N, WILSON Janet $A$. Is Laryngopharyngeal Reflux Related to Functional Dysphonia? Annals of Cology Rhinology \& Laryngology 2007; 116: 24-9.

15. Ylitalo R, Ramel S. Extraesophageal reflux in patients with contact granuloma: a prospective controlled study. Ann Otol Rhinol Laryngol. 2002; 111: 441-6.

16. Belafsky PC, Postma GN, Koufman JA. The validity and reliability of the Reflux Finding Score (RFS). Laryngoscope. 2001; 111: 1313-7.

17. Poelmans J, Feenstra L, Demedts I et al. The yield of upper gastrointestinal endoscopy in patients with suspected reflux-related chronic ear, nose and throat symptoms. Am J Gastroenterol 2004; 99: 1419-26.

18. Hopkins C, Yousaf U, Pedersen M. Acid reflux treatment for hoarseness [protocol]. Cochrane Database Syst Rev. 2005(3) Accession № 00075320-10000000-03935. 